\title{
Naptumomab Estafenatox: Targeted Immunotherapy with a Novel Immunotoxin
}

\author{
Tim Eisen • Gunnar Hedlund • Göran Forsberg • \\ Robert Hawkins
}

Published online: 21 January 2014

(C) The Author(s) 2014. This article is published with open access at Springerlink.com

\begin{abstract}
Improvement of cancer therapy by introducing new concepts is still urgent even though there have been major advancements lately. Immunotherapy is well on the way to becoming an established tool in the cancer treatment armory. It seems that a combination of (1) activation of immune effector cells and selective targeting of them to tumors and (2) the inhibition of immune suppression often induced by the tumor itself are necessary to achieve the therapeutic goal. The immunotoxin naptumomab estafenatox was developed in an effort to activate and target the patient's own T cells to their tumor, by fusing a superantigen $(\mathrm{SAg})$ variant that activates $\mathrm{T}$ lymphocytes to the Fab moiety of a tumor-reactive monoclonal antibody. Naptumomab estafenatox targets the 5T4 tumor antigen, a $72-\mathrm{kDa}$ oncofetal trophoblast protein expressed on many carcinomas, including renal cell carcinoma. The therapeutic effect is associated with activation of SAg-binding T cells. The SAg-binding T lymphocytes expand, differentiate to effector cells, and infiltrate the tumor. The therapeutic efficacy is most likely related to the dual mechanism of tumor cell killing: (1) direct lysis by cytotoxic $\mathrm{T}$ lymphocytes of tumor cells expressing the antigen recognized by the antibody moiety of the fusion protein and (2) secretion of cytokines eliminating antigen-negative tumor cell
\end{abstract}

This article is part of the Topical Collection on Evolving Therapies

T. Eisen $(\bowtie)$

Cambridge University Health Partners, Addenbrooke's Hospital,

Cambridge, UK

e-mail: tgqe2@medschl.cam.ac.uk

G. Hedlund $\cdot$ G. Forsberg

Active Biotech AB, Lund, Sweden

R. Hawkins

The Christie NHS Foundation Trust, Manchester, UK

R. Hawkins

University of Manchester, Manchester, UK variants. Naptumomab estafenatox has been clinically tested in a range of solid tumors with focus on renal cell carcinoma. This review looks at the clinical experience with the new immunotoxin and its potential.

Keywords Immunotherapy · Immunotoxin · Tumor-targeted superantigen $\cdot 5 \mathrm{~T} 4$ tumor antigen $\cdot$ Carcinoma $\cdot$ Kidney cancer $\cdot$ Renal cell carcinoma $\cdot$ Evolving therapies . Naptumomab estafenatox

\section{Introduction}

The therapeutic landscape for many malignancies is rapidly changing. Among the important developments of recent years is the rise of therapies activating or releasing the power of immunological mechanisms. Older immune therapies against solid malignancies have proved toxic and only modestly active in clinical practice. However, a deeper understanding of tumor immunology has led to recent successes, including the FDA approval of sipuleucel-T to treat prostate cancer as well as ipilimumab to treat malignant melanoma. Although certain cancers have been identified as being susceptible to immune-based interventions, new evidence indicates that immunotherapies might become a general therapeutic tool in the treatment of cancer. It seems that a combination of (1) activation of immune effector cells and selective targeting of them to tumors and (2) the inhibition of immune suppression often induced by the tumor itself are necessary to achieve the therapeutic goal. In an effort to activate and target the patient's own $\mathrm{T}$ cells to their tumor, the immunotoxin naptumomab estafenatox was developed and has so far been clinically tested in a range of solid tumors with focus on renal cell carcinoma. This report provides an overview of the clinical experience and the status of development of the naptumomab estafenatox (ABR-217620; ANYARA). 


\section{Naptumomab Estafenatox-a Tumor-Targeted Superantigen}

The tumor-targeted superantigen (TTS) concept uses a unique approach to target large numbers of $\mathrm{T}$ cells to tumors $[1,2 \cdot 3]$ and utilizes a distinct type of immunotoxin. $\mathrm{T}$ lymphocytes are very effective in eliminating some experimental tumors. However, in humans, tumorspecific $\mathrm{T}$ cells often occur in low numbers or may be suppressed. Therefore, their numbers are insufficient to interfere with established growing cancer. In the TTS concept, bacterial superantigens (SAgs), the most potent known activators of human T cells, are used to activate and recruit large numbers of $\mathrm{T}$ cells to the targeted tumors.

The wild-type SAg staphylococcal enterotoxin A (SEA) binds effectively to MHC class $\mathrm{II}^{+}$accessory cells and activates both $\mathrm{CD}^{+}$and $\mathrm{CD} 8^{+} \mathrm{T}$ cells $[4,5]$. MHC class $\mathrm{II}^{+}$cells are killed by cytotoxic T lymphocytes (CTLs) when targeted by SEA [6-9]. A tumor-specific SAg is created by fusing the SAg to the Fab moiety of a tumor-reactive monoclonal antibody $(\mathrm{mAb})$. Fab-SAg fusion proteins have been successfully used to cure mice with established experimental tumors. The therapeutic effect was associated throughout the tumor with a massive infiltration of $\mathrm{T}$ cells which actively produced tumoricidal cytokines such as tumor necrosis factor $\alpha$ and interferon (IFN)- $\gamma$. The therapeutic efficacy is most likely related to the dual mechanism of tumor cell killing: direct lysis of tumor cells expressing the antigen recognized by the antibody moiety of the fusion protein and secretion of cytokines eliminating antigen-negative tumor cell variants (Fig. 1).

The TTS therapeutic proteins, including naptumomab estafenatox, are typically used in cycles of four to five oncedaily intravenous injections. In the first phase of a cycle, the $\mathrm{T}$ lymphocytes are activated and differentiate into effector cells, which later in the cycle localize to the tumor and mediate their antitumor functions (Fig. 1). This treatment schedule can be repeated and is easily combined with other anticancer drug modalities.

The early clinical trials with TTS were performed using wild-type SEA as the T-cell activator $[10,11]$. However, this fusion protein (nacolomab tafenatox) was associated with a most potent T-cell activation and therefore it was necessary to give the intravenous doses at only a few nanograms per kilogram to avoid unacceptable toxicity. In addition, the dose had to be individualized on the basis of preformed anti-SAg (anti-SEA) antibodies. Wild-type SAgs bind to MHC class II cell membrane proteins, and the complexes subsequently bind to T-cell receptors (TCRs) containing certain $\mathrm{V} \beta$ sequences. After proliferation and differentiation, these $\mathrm{T}$ lymphocytes infiltrate the tumor tissue and are activated in the tumor owing to TCR binding to the SAgs of the TTS protein. The activated $\mathrm{T}$ cells produce cytokines, mainly of the $\mathrm{T}_{\mathrm{h}} 1$ type, e.g., IL-2

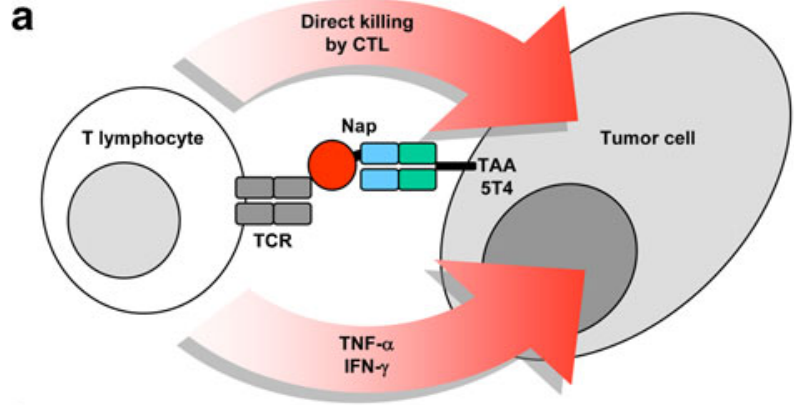

b

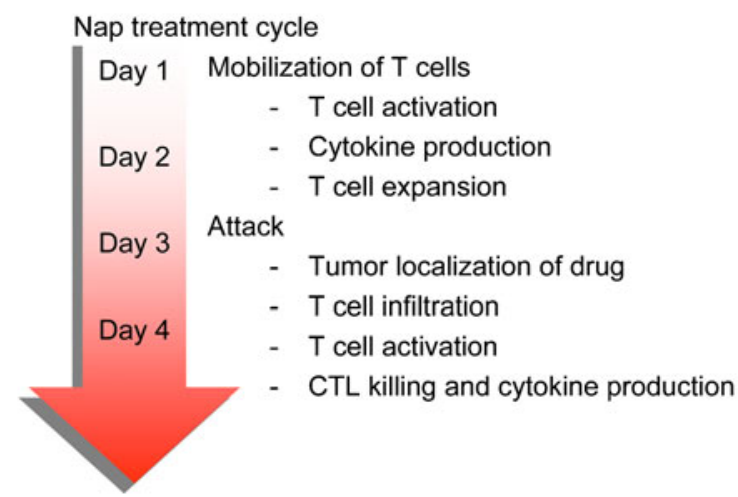

C

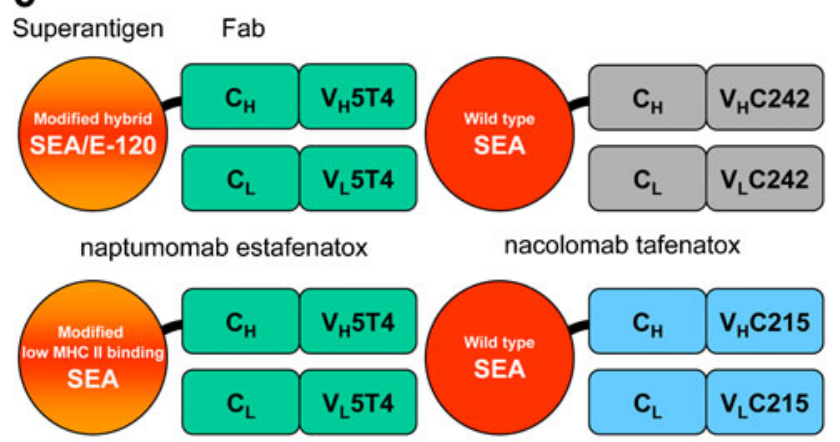

anatumomab mafenatox C215Fab-SEA; used in mouse models

Fig. 1 a Mechanism of action of naptumomab estafenatox (Nap). b Immune/drug functions during a naptumomab estafenatox (Nap) treatment cycle. c Different fusion proteins. The tumor-targeted superantigens are naptumomab estafenatox, anatumomab mafenatox, nacolomab tafenatox, and C215-staphylococcal enterotoxin A (SEA). CTL cytotoxic T lymphocyte, $I F N$ interferon, TAA tumor-associated antigen, TCR T-cell receptor, $T N F$ tumor necrosis factor

and IFN- $\gamma$, and perform direct perforin-dependent tumor killing. To achieve maximal targeted antitumor effects, a balanced relationship between the binding affinities of the three functional binding sites in the TTS is required. The desire to mimic the T-effector cell (e.g., the CTL) binding via its TCR to the MHC/tumor peptide target on a tumor target cell drove the development of naptumomab estafenatox, which showed distinct characteristics regarding binding to the tumor antigen (5T4), the TCR, and the MHC class II proteins [12••, 13]. It is currently hypothesized that an optimal TTS should have very high affinity for the tumor antigen, low affinity for TCR, and very low affinity for MHC class II proteins. Naptumomab 
estafenatox binds to the 5T4 tumor antigen with $K_{\mathrm{D}}$ of approximately $1 \mathrm{nM}$. SEA/E-120, the SAg part of naptumomab estafenatox, has been characterized in detail and has been found to primarily bind and operate through TCR TRBV7-9 with a binding constant of $0.48 \mu \mathrm{M}$ and an affinity for MHC class II proteins of $51 \mu \mathrm{M}$.

A fusion protein consisting of the Fab moiety of the $\mathrm{mAb}$ 5T4 fused to SEA with a point mutation decreasing MHC class II protein binding (associated with decreased toxicity), anatumomab mafenatox [14], a predecessor of naptumomab estafenatox, was investigated in clinical phase 1 and phase 2 trials with encouraging results $[15,16 \bullet]$. However, this fusion protein was also associated with some of the drawbacks seen with nacolomab tafenatox, particularly the need to give low doses as well as individualized dosing based on preformed anti-SAg (anti-SEA) antibodies. The novel genetically modified immunotoxin naptumomab estafenatox displays strong affinity for the $5 \mathrm{~T} 4$ tumor antigen, on the order of $1 \mathrm{nM}$, and is approximately $50 \%$ more efficient than anatumomab mafenatox in killing human 5T4-positive tumor cells in vitro [12••]. Naptumomab estafenatox does induce an MHC class II and dose dependent proliferation of human lymphocytes, but only at 50 -fold and more than $10^{3}$-fold higher concentrations than anatumomab mafenatox and nacolomab tafenatox, respectively $[12 \bullet \bullet]$. Furthermore, preformed human anti-SAg antibodies did not recognize the functional regions in the SAg part of naptumomab estafenatox to the same extent as in the predecessor construct [12・•]. Thus, even though naptumomab estafenatox has very potent antitumor properties, it contains an SAg moiety without classic SAg activity. The MHC class II binding capacity is minimal and the T-cell proliferation induced by naptumomab estafenatox has a median effective concentration more than $10^{3}$ times higher than those of conventional wild-type SAgs, resulting in dramatically reduced toxicity. Furthermore, the levels of target epitopes for naturally occurring anti-SAg antibodies were reduced and dosing of naptumomab estafenatox was therefore considered to be independent of normal baseline levels of anti-SAg antibodies.

Naptumomab estafenatox targets the 5T4 tumor antigen, a $72-\mathrm{kDa}$ oncofetal trophoblast protein originally isolated from placenta and characterized by the use of the murine IgG1 $\mathrm{mAb}$ $5 \mathrm{~T} 4[17,18]$. Expression of 5T4 antigen has been shown to influence adhesion, cytoskeletal organization, and motility, properties which might account for its association with poorer clinical outcome in some cancers [19]. A general screen by immunohistochemistry on frozen sections showed that many different carcinomas express the 5T4 antigen [20-27] (Table 1), and very often the 5T4 antigen levels appear to be higher in more advanced stages of cancer. Studies have shown a homogenous reactivity, which in most cases is moderate to strong, in more than $95 \%$ of non-small-cell lung cancer
Table $15 \mathrm{~T} 4$ reactivity in a variety of tumor types Cancer

\begin{tabular}{|c|c|c|c|}
\hline Cancer & Stage/Type & Reactivity & Conclusions \\
\hline Breast & Mixed & $56 / 63$ & $\begin{array}{l}85-95 \% \text { of patients express the } \\
5 \mathrm{~T} 4 \text { antigen. }\end{array}$ \\
\hline \multirow[t]{5}{*}{ Cervix } & Mixed & $5 / 5$ & \multirow{5}{*}{$\begin{array}{l}85-90 \% \text { of patients express the } \\
5 \mathrm{~T} 4 \text { antigen. }\end{array}$} \\
\hline & I & $22 / 25$ & \\
\hline & II & $22 / 26$ & \\
\hline & III & $9 / 10$ & \\
\hline & IV & $5 / 6$ & \\
\hline \multirow[t]{5}{*}{ Colorectal } & Mixed & $11 / 22$ & \multirow{5}{*}{$\begin{array}{l}40-50 \% \text { of patients express the } \\
5 \mathrm{~T} 4 \text { antigen. In Dukes stage } \\
\text { D patients, more than } 70 \% \\
\text { over-express the antigen. }\end{array}$} \\
\hline & A & $2 / 8$ & \\
\hline & B & $7 / 34$ & \\
\hline & $\mathrm{C}$ & $13 / 21$ & \\
\hline & $\mathrm{D}$ & $7 / 9$ & \\
\hline \multirow[t]{5}{*}{ Gastric } & Mixed & $41 / 93$ & \multirow{5}{*}{$\begin{array}{l}\text { Approximately } 50 \% \text { of patients } \\
\text { express the } 5 \mathrm{~T} 4 \text { antigen on } \\
\text { tumor cells. Most samples with } \\
\text { 5T4 negative tumor cells have } \\
\text { 5T4 positive tumor stroma } \\
\text { [25]. }\end{array}$} \\
\hline & I & $1 / 2$ & \\
\hline & II & $1 / 4$ & \\
\hline & III & $1 / 1$ & \\
\hline & IV & $12 / 20$ & \\
\hline NSCLC & Mixed & $259 / 261$ & $\begin{array}{l}99 \% \text { of patients express the } \\
5 \mathrm{~T} 4 \text { antigen. }\end{array}$ \\
\hline \multirow[t]{5}{*}{ Ovarian } & Mixed & $4 / 7$ & \multirow{5}{*}{$\begin{array}{l}\text { More than } 70 \% \text { of patients } \\
\text { express the } 5 \mathrm{~T} 4 \text { antigen.. } \\
\text { For stage IV patients, } \\
90-95 \% \text { of the patients } \\
\text { are } 5 \mathrm{~T} 4 \text { positive. }\end{array}$} \\
\hline & I & $2 / 10$ & \\
\hline & II & $4 / 57$ & \\
\hline & III & $21 / 29$ & \\
\hline & IV & $24 / 26$ & \\
\hline Pancreatic & Mixed & $23 / 23$ & $\begin{array}{l}100 \% \text { of patients express the } \\
5 \mathrm{~T} 4 \text { antigen. }\end{array}$ \\
\hline Prostate & Mixed & $26 / 26$ & $\begin{array}{l}100 \% \text { of patients express the } \\
5 \mathrm{~T} 4 \text { antigen. }\end{array}$ \\
\hline \multirow[t]{3}{*}{ Renal } & Clear cell & $215 / 222$ & \multirow{3}{*}{$\begin{array}{l}>95 \% \text { of patients express the } \\
5 \mathrm{~T} 4 \text { antigen. }\end{array}$} \\
\hline & Papillary & $18 / 18$ & \\
\hline & Mixed & $36 / 37$ & \\
\hline
\end{tabular}

(NSCLC), pancreatic cancer (PC), and renal cell cancer (RCC). In many cases, tumor stromal reactivity has been observed in addition to tumor cell reactivity. Outside the placenta, 5T4 normal tissue reactivity is considered to be most limited and there is no evidence of circulating 5T4 antigen. Naptumomab estafenatox with the selectivity of the $5 \mathrm{~T} 4 \mathrm{mAb}$ has been in clinical development primarily for the indications of NSCLC and RCC. It targets a tumor antigen highly expressed on tumor cells, with very low expression on normal tissue and without soluble antigen interference.

\section{Naptumomab Estafenatox-Phase 1 Studies}

Naptumomab estafenatox was developed in parallel with the initial clinical studies with its predecessor anatumomab mafenatox and has been evaluated in phase 1 trials in NSCLC, PC, and RCC patients [28••, 29]. Altogether, 52 
patients were investigated in the monotherapy (MONO) trial and the combination therapy (COMBO) trial with docetaxel. The primary aim was to establish the maximum tolerated dose of naptumomab estafenatox. The secondary objectives were to determine the safety profile, pharmacokinetic parameters, immunological response, and effects on tumor disease. The MONO study was conducted in Norway, the UK, and the USA. The main inclusion criteria were histologically or cytologically confirmed disease, which was refractory to available standard therapies. Patients had to have an Eastern Cooperative Oncology Group performance status of 0 or 1 and life expectancy of more that 3 months. Three different indications were investigated, consisting of 20 cases of NSCLC, 11 cases of RCC, and eight cases of PC. The starting dosage was $500 \mathrm{ng} / \mathrm{kg} /$ day administrated as a 5 -min bolus injection and naptumomab estafenatox was given for five consecutive days. Six dose-limiting toxicities were recorded, and the highest dosage investigated was $27.4 \mu \mathrm{g} / \mathrm{kg} / \mathrm{day}$. Patients with stable disease or response were considered for treatment with a second 5-day cycle, unless they had experienced a dose-limiting toxicity. The most notable side effects were fever, nausea, and rigors, and these were easily managed. It was concluded that the side effects were not dependant on the anti-SAg (anti-SEA/E-120) antibody titers. The recommended phase 2 dosage was set at $15 \mu \mathrm{g} / \mathrm{kg} /$ day for patients with RCC and $22 \mu \mathrm{g} / \mathrm{kg} /$ day for patients with NSCLC and PC.

As part of the immune activation, increased systemic levels of IL-2, IFN- $\gamma$, and IL-10 were observed $3 \mathrm{~h}$ after naptumomab estafenatox treatment, and there was a correlation between the dose and cytokine induction. We have evaluated the cytokine responses as a surrogate for a beneficial pharmacologic effect [16 ${ }^{\bullet}$ in all patients from the MONO and COMBO studies. Plasma IL-2 levels were dose-dependently increased $3 \mathrm{~h}$ after injection of naptumomab estafenatox in the first treatment cycle [28••]. RCC patients had larger increases than NSCLC or PC patients. The IL-2 response declined in the later parts of the treatment cycle, showing only marginally elevated IL-2 levels on days 4 and 5. Plasma IFN- $\gamma$ levels were also dose-dependently increased $3 \mathrm{~h}$ after TTS injection. The IFN- $\gamma$ response declined only slightly as the treatment cycle progressed, and unlike IL-2, high IFN- $\gamma$ levels were also recorded on days 4 and 5. Plasma IL-10 levels were dosedependently increased $3 \mathrm{~h}$ after injection of naptumomab estafenatox and continued to increase as the treatment cycle progressed. One may speculate that a powerful immune activation stimulates inhibitory responses, such as IL-10 production, similar to that seen as a vigorous antivirus response is naturally downregulated.

A mechanistically important finding was that the SAg reactive population of T cells expressing the TCR TRBV7-9 was selectively expanded after naptumomab estafenatox treatment and returned to normal levels at day 28. The anti-SAg (anti-SEA/E-120) levels were stable or only moderately increased in approximately $50 \%$ of the patients after one cycle, and human anti-mouse antibody levels were very low.

A major objective in the development of the TTS fusion proteins has been a short systemic exposure to provide high tumor targeting capability combined with acceptable toxicity. The pharmacokinetics of naptumomab estafenatox show a small volume of distribution (about $0.14 \mathrm{~L} / \mathrm{kg}$ ) and a low clearance (about $0.13 \mathrm{~L} / \mathrm{h} / \mathrm{kg}$ ). The mean terminal half-life was determined to be $1.4 \mathrm{~h}$. No time dependency was seen in the pharmacokinetics in the first treatment cycle, with similar plasma concentrations on day 1 and day 5. Furthermore, no dose dependency in the pharmacokinetics was indicated at doses of $10 \mu \mathrm{g} / \mathrm{kg}$ and greater and no effect of gender or disease indication in the pharmacokinetic parameters of naptumomab estafenatox was seen. During the first treatment cycle no significant effect of anti-SAg (antiSEA/E-120) antibody levels on pharmacokinetic parameters was seen .

Efficacy was analyzed by CT scan. Several patients in the MONO study had tumor shrinkage, not amounting to formal Response Evaluation Criteria in Solid Tumors partial responses. The median overall survival times were 15.8 months for the NSCLC patients, 26.2 months for the RCC patients, and 5.2 months for the PC patients. Although these studies were not controlled and a bias in patient selection cannot be excluded, the median overall survival for the NSCLC and RCC patients was encouraging. It should be noted that these early studies were performed prior to the widespread use of antiangiogenic agents in the treatment of RCC.

An open-label dose-escalation study of naptumomab estafenatox in combination with docetaxel (COMBO) was conducted in Denmark, Russia, and the USA [28••]. In this COMBO study, 13 patients with advanced NSCLC were treated. The primary end point was to describe the side effect profile of the combination treatment and establish the maximum tolerated dose of naptumomab estafenatox in combination with docetaxel. Secondary end points included pharmacokinetics, immunological responses, changes in anti-SAg (anti-SEA/E-120) levels, and effects on tumor disease. Patients were treated with a bolus injection of naptumomab estafenatox in $5 \mathrm{~min}$ for four consecutive days followed by $75 \mathrm{mg} / \mathrm{m}^{2}$ docetaxel on day 5 . Three weeks later this procedure was repeated. Three patients received naptumomab estafenatox at a dosage of $10 \mu \mathrm{g} / \mathrm{kg} / \mathrm{day}$, three patients received it at a dosage of $16.5 \mu \mathrm{g} / \mathrm{kg} /$ day, and seven patients received it at a dosage of $22 \mu \mathrm{g} / \mathrm{kg} /$ day. No synergistic toxicities were observed. The best overall responses were two patients with partial response, five patients with stable disease, and three patients with progressive disease, and three patients were not evaluable. Biopsy specimens were taken from two patients on day 3 of the second cycle. In both patients tumor 5T4 expression and marked T-cell infiltration of the 
metastases were confirmed. One of these patients had a partial response. The median survival for the patients was 9.3 months. Formation of antibody against SEA/E-120 was low in these patients. Anti-SEA/E-120 levels were compared after the first treatment cycle in the COMBO study with those in the MONO study, and the comparison showed that docetaxel appeared to reduce anti-SAg antibody production when it was given 1 day after the last injection in a treatment cycle with naptumomab estafenatox. Since the avoidance of hightiter neutralizing antibodies would be advantageous for multicycle treatment with naptumomab estafenatox, combinations with docetaxel or other inhibitors of anti-SAg antibody production might be beneficial.

\section{Naptumomab Estafenatox-Phase 2/3 Studies and Baseline Biomarkers for RCC Patient Selection}

In preclinical models, IFN- $\alpha$ had been shown to enhance and sustain TTS-induced CTL activity when given in combination [30]. This observation led to a randomized phase $2 / 3$ trial of naptumomab estafenatox plus IFN- $\alpha$ versus IFN- $\alpha$ conducted in 513 patients with advanced RCC. The patients were recruited between March 2007 and May 2009 and randomized 1:1 in an open-label study to receive naptumomab estafenatox plus IFN- $\alpha$ or IFN- $\alpha$ : $15 \mu \mathrm{g} / \mathrm{kg}$ naptumomab estafenatox was given intravenously in three cycles of four once-daily injections plus IFN- $\alpha$ (9 MU subcutaneously three times weekly) or the same dose and schedule of IFN- $\alpha$ monotherapy alone. The primary end point was overall survival. The secondary end points were progression-free survival, response rate, and safety.

The first set of results was presented at the 2013 American Society of Clinical Oncology annual meeting [31]. Although the primary end point of overall survival (hazard ratio (HR) of 1.08) was not reached, the secondary endpoint of progressionfree survival showed a HR of 0.92. Exploratory endpoints of anti-SAg (anti-SEA/E-120), a biomarker for drug exposure, and IL-6, a biomarker for immune responsiveness, were assessed as predictive biomarkers. In contrast to the results of earlier studies, significantly increased baseline anti-SAg (anti-SEA/E-120) antibody levels were detected in certain territories. In a subgroup of patients having below median baseline levels of anti-SAg (anti-SEA/E-120) and IL-6, prolonged progression-free survival $(\mathrm{HR}=0.62, p=0.016)$ and overall survival $(\mathrm{HR}=0.59, p=0.020)$ were achieved. Naptumomab estafenatox was well tolerated. Most of the adverse events resulting from treatment with naptumomab estafenatox related to increased levels of cytokines and are expected as part of the mechanism of action. Pyrexia, vomiting, nausea, chills, and back pain were commoner after treatment with naptumomab estafenatox.

A detailed analysis of the results is ongoing and the result will therefore not be discussed further in this review. As previously described, naptumomab estafenatox is a fusion protein containing an engineered hybrid of Staphylococcus entertoxins A and E (SEA/E-120). Although SEA/E-120 has been engineered to express a minimum of antigenic epitopes in the wild-type SAgs recognized by patient antibodies at the baseline [12••], certain patients may have elevated levels owing to cross-reactivity to previously encountered wildtype Staphylococcus enterotoxins, e.g., through infections from Staphylococcus aureus. The results from the phase 1 studies showed that baseline plasma anti-SAg (anti-SEA/E120) levels were low in most patients and indicated that the exposure of clinically relevant doses $(12 \mu \mathrm{g} / \mathrm{kg}$ or more $)$ of naptumomab estafenatox was independent of the low levels of baseline antibodies. In the phase $2 / 3$ study of RCC patients, the number of patients was vastly expanded and the countries in which they were recruited changed from the phase 1 studies. Increased baseline anti-SAg (anti-SEA/E-120) antibody levels were detected in certain territories, predicting suboptimal exposure [31], which may affect drug activity and antitumor efficacy. Therefore, anti-SAg (anti-SEA/E-120) seems to be a baseline biomarker for exposure important for the selection of patients for treatment with naptumomab estafenatox.

\section{Conclusion}

Naptumomab estafenatox has therapeutic potential in tumors expressing the 5T4 antigen. Clinical development of naptumomab estafenatox is now focused on the identified patient subset of RCC and studies using naptumomab estafenatox add-on treatment with established tyrosine kinase inhibitors in the first or second line. Furthermore, as data accumulate regarding the necessity of using combinations of immunotherapies to reach full effect [32•], naptumomab estafenatox might require the modulation of immune checkpoints with, e.g., ipilimumab or nivolumab, for optimal activity.

\section{Compliance with Ethics Guidelines}

Conflict of Interest Tim Eisen holds stock in Astra Zeneca and has been a consultant for Bayer, Pfizer, Roche, GSK and AVEO.

Gunnar Hedlund has received stock options from Active Biotech. Göran Forsberg has received stock options from Active Biotech. Robert Hawkins declares that he has no conflict of interest.

Human and Animal Rights and Informed Consent This article is a review and does not contain any new studies with human or animal subjects performed by any of the authors.

Open Access This article is distributed under the terms of the Creative Commons Attribution License which permits any use, distribution, and reproduction in any medium, provided the original author(s) and the source are credited. 


\section{References}

Papers of particular interest, published recently, have been highlighted as:

- Of importance

•- Of major importance

1. Dohlsten M, Hedlund G, Akerblom E, Lando PA, Kalland T. Monoclonal antibody-targeted superantigens: a different class of anti-tumor agents. Proc Natl Acad Sci U S A. 1991;88:9287-91.

2. Dohlsten M, Abrahmsen L, Bjork P, Lando PA, Hedlund G, et al. Monoclonal antibody-superantigen fusion proteins: tumor-specific agents for T-cell-based tumor therapy. Proc Natl Acad Sci U S A. 1994;91:8945-9. This article describes the pre-clinical background for the Tumor Targeted Superantigen concept.

3. Dohlsten M, Hansson J, Ohlsson L, Litton M, Kalland T. Antibodytargeted superantigens are potent inducers of tumor-infiltrating $\mathrm{T}$ lymphocytes in vivo. Proc Natl Acad Sci U S A. 1995;92:9791-5.

4. Carlsson R, Fischer H, Sjogren HO. Binding of staphylococcal enterotoxin A to accessory cells is a requirement for its ability to activate human T cells. J Immunol. 1988;140:2484-8.

5. Fischer H, Dohlsten M, Lindvall M, Sjogren HO, Carlsson R. Binding of staphylococcal enterotoxin A to HLA-DR on B cell lines. J Immunol. 1989;142:3151-7.

6. Hedlund G, Dohlsten M, Lando PA, Kalland T. Staphylococcal enterotoxins direct and trigger CTL killing of autologous HLA$\mathrm{DR}+$ mononuclear leukocytes and freshly prepared leukemia cells. Cell Immunol. 1990;129:426-34.

7. Hedlund G, Dohlsten M, Petersson C, Kalland T. Superantigenbased tumor therapy: in vivo activation of cytotoxic T cells. Cancer Immunol Immunother. 1993;36:89-93.

8. Dohlsten M, Lando PA, Hedlund G, Trowsdale J, Kalland T. Targeting of human cytotoxic T lymphocytes to MHC class II-expressing cells by staphylococcal enterotoxins. Immunology. 1990;71:96-100.

9. Dohlsten M, Hedlund G, Kalland T. Staphylococcal-enterotoxindependent cell-mediated cytotoxicity. Immunol Today. 1991;12: 147-50.

10. Giantonio BJ, Alpaugh RK, Schultz J, McAleer C, Newton DW, et al. Superantigen-based immunotherapy: a phase I trial of PNU214565, a monoclonal antibody-staphylococcal enterotoxin A recombinant fusion protein, in advanced pancreatic and colorectal cancer. J Clin Oncol. 1997;15:1994-2007.

11. Alpaugh RK, Schultz J, McAleer C, Giantonio BJ, Persson R, et al. Superantigen-targeted therapy: phase I escalating repeat dose trial of the fusion protein PNU-214565 in patients with advanced gastrointestinal malignancies. Clin Cancer Res. 1998;4:1903-14.

12.• Forsberg G, Skartved NJ, Wallen-Ohman M, Nyhlen HC, Behm K, et al. Naptumomab estafenatox, an engineered antibodysuperantigen fusion protein with low toxicity and reduced antigenicity. J Immunother. 2010;33:492-9. This article describes the molecular characteristics and preclinical efficacy studies of naptumomab estafenatox.

13. Hedlund G, Eriksson H, Sundstedt A, Forsberg G, Jakobsen BK, Pumphrey N, et al. The tumor targeted superantigen ABR-217620 selectively engages TRBV7-9 and exploits TCR-pMHC affinity mimicry in mediating T cell cytotoxicity. PLoS One. 2013;8(10): e79082.

14. Forsberg G, Ohlsson L, Brodin T, Bjork P, Lando PA, et al. Therapy of human non-small-cell lung carcinoma using antibody targeting of a modified superantigen. Br J Cancer. 2001;85:129-36.

15. Cheng JD, Babb JS, Langer C, Aamdal S, Robert F, et al. Individualized patient dosing in phase I clinical trials: the role of escalation with overdose control in PNU-214936. J Clin Oncol. 2004;22:602-9.
16. Shaw DM, Connolly NB, Patel PM, Kilany S, Hedlund G, et al. A phase II study of a 5T4 oncofoetal antigen tumour-targeted superantigen (ABR-214936) therapy in patients with advanced renal cell carcinoma. Br J Cancer. 2007;96:567-74. This article presents the results of the phase 2 study with the predecessor compound anatumomab mafenatox in RCC exploring early efficacy and IL-2 as a pharmacodynamic biomarker.

17. Hole N, Stern PL. A $72 \mathrm{kD}$ trophoblast glycoprotein defined by a monoclonal antibody. Br J Cancer. 1988;57:239-46.

18. Hole N, Stern PL. Isolation and characterization of 5T4, a tumourassociated antigen. Int J Cancer. 1990;45:179-84.

19. Southgate TD, McGinn OJ, Castro FV, Rutkowski AJ, Al-Muftah $\mathrm{M}$, et al. CXCR4 mediated chemotaxis is regulated by $5 \mathrm{~T} 4$ oncofetal glycoprotein in mouse embryonic cells. PLoS ONE. 2010;5:e9982.

20. Starzynska T, Marsh PJ, Schofield PF, Roberts SA, Myers $\mathrm{KA}$, et al. Prognostic significance of 5T4 oncofetal antigen expression in colorectal carcinoma. Br J Cancer. 1994;69:899 902.

21. Southall PJ, Boxer GM, Bagshawe KD, Hole N, Bromley M, et al. Immunohistological distribution of 5T4 antigen in normal and malignant tissues. Br J Cancer. 1990;61:89-95.

22. Jones H, Roberts G, Hole N, McDicken IW, Stern P. Investigation of expression of 5T4 antigen in cervical cancer. Br J Cancer. 1990;61:96-100.

23. Naganuma H, Kono K, Mori Y, Takayoshi S, Stern PL, et al. Oncofetal antigen 5T4 expression as a prognostic factor in patients with gastric cancer. Anticancer Res. 2002;22: 1033-8.

24. Starzynska T, Rahi V, Stern PL. The expression of 5T4 antigen in colorectal and gastric carcinoma. Br J Cancer. 1992;66: 867-9.

25. Starzynska T, Wiechowska-Kozlowska A, Marlicz K, Bromley $\mathrm{M}$, Roberts SA, et al. 5T4 oncofetal antigen in gastric carcinoma and its clinical significance. Eur J Gastroenterol Hepatol. 1998;10:479-84.

26. Wrigley E, McGown AT, Rennison J, Swindell R, Crowther D, et al. 5T4 oncofetal antigen expression in ovarian carcinoma. Int J Gynecol Cancer. 1995;5:269-74.

27. Connor ME, Stern PL. Loss of MHC class-I expression in cervical carcinomas. Int J Cancer. 1990;46:1029-34.

28.• Borghaei H, Alpaugh K, Hedlund G, Forsberg G, Langer C, et al. Phase I dose escalation, pharmacokinetic and pharmacodynamic study of naptumomab estafenatox alone in patients with advanced cancer and with docetaxel in patients with advanced non-small-cell lung cancer. J Clin Oncol. 2009;27:4116-23. This article describes the phase 1 results comprising safety, early efficacy and pharmacodynamic responses with naptumomab estafentox in patients with RCC, NSCLC and pancreatic cancer.

29. Robinson MK, Alpaugh RK, Borghaei H. Naptumomab estafenatox: a new immunoconjugate. Expert Opin Biol Ther. 2010;10:273-9.

30. Sundstedt A, Celander M, Hedlund G. Combining tumor-targeted superantigens with interferon-alpha results in synergistic anti-tumor effects. Int Immunopharmacol. 2008;8:442-52.

31. Hawkins RE, Gore ME, Shparyk Y, Bondar V, Gladkov O, Ganev $\mathrm{T}$, et al. A randomized phase II/III study of naptumomab estafenatox plus IFN- $\alpha$ versus IFN- $\alpha$ in advanced renal cell carcinoma. J Clin Oncol. 2013;31(15 Suppl):3073.

31. Nirschl CJ, Drake CG. Molecular pathways: co-expression of immune checkpoint molecules: signaling pathways and implications for cancer immunotherapy. Clin Cancer Res. 2013;19:4917-24. This article emphasizes the importance of controlling the immune checkpoints to achieve effective immune therapy, implicating a combinatory benefit with naptumomab estafenatox. 